\title{
NILPOTENT ELEMENTS IN RINGS OF INTEGRAL REPRESENTATIONS
}

\author{
IRVING REINER
}

1. Introduction. Let $G$ be a finite group, and let $R$ be a discrete valuation ring of characteristic zero, with maximal ideal $P=\pi R$, and whose residue class field $\bar{R}=R / P$ has characteristic $p \neq 0$. By an $R G$-module we mean always a left $R G$-module which is finitely generated over $R$, though not necessarily $R$-torsionfree. Assume that the Krull-Schmidt theorem is valid for $R G$-modules; this is certainly the case when $R$ is complete, or when $R$ is a valuation ring in an algebraic number field which is a splitting field for $G$.

In a recent paper [4] we introduced the integral representation ring, denoted by $A(R G)$, defined as the additive group generated by the symbols $\{M\}$, one for each isomorphism class of $R$-torsionfree $R G$ modules, with relations $\{M \oplus N\}=\{M\}+\{N\}$. Multiplication in $A(R G)$ is defined by taking tensor products of modules.

The question arises as to whether the commutative ring $A(R G)$ contains any nonzero nilpotent elements. This is of special interest in view of recent results of Green [2] and O'Reilly [3], who showed that if $k$ is a field of characteristic $p$, and if $G$ has a cyclic $p$-Sylow subgroup, then $A(k G)$ has no nonzero nilpotent elements.

In contradistinction to this, we proved in [4]:

THEOREM 1. Let $G^{*}$ be a cyclic group of order $n$, and suppose that the Krull-Schmidt theorem holds for $R G^{*}$-modules. Assume that $n \in P^{2}$, and if $2 \in P$ assume further that $n \in 2 P$. Then $A\left(R G^{*}\right)$ contains at least one nonzero nilpotent element.

The aim of the present note is to establish the following generalization.

THEOREM 2. Suppose that the group $G$ contains a cyclic subgroup $G^{*}$ satisfying the hypotheses of Theorem 1, and assume that the KrullSchmidt theorem holds for $R G$-modules. Then $A(R G)$ contains at least one nonzero nilpotent element.

We shall use the following notation. For $M$ an $R G$-module, set $\bar{M}=M / P M$. Denote by $M_{H}$ the $R H$-module obtained from $M$ by

Presented to the Society, November 23, 1964; received by the editors November 21, 1964. 
restriction of operators, where $H$ is a subgroup of $G$. For $N$ an $R H$ module, let $N^{G}$ denote the induced $R G$-module defined by

$$
N^{G}=R G \otimes_{R H} N .
$$

The trivial $R G$-module is $R$ itself, on which each $g \in G$ acts as identity operator. If $M, N$ are $R G$-modules, the notation $M \mid N$ means that $M$ is isomorphic to an $R G$-direct summand of $N$.

As general reference for the techniques and definitions used in this note, we refer the reader to [1].

2. Preliminaries to the proof. Suppose hereafter that the hypotheses of Theorem 2 are satisfied, so that $G$ contains a cyclic subgroup $G^{*}$ of order $n$. If $p$ is the unique rational prime contained in $P$, then the assumptions about $n$ readily imply that $p^{e} \mid n$, where

$$
e= \begin{cases}2, & p=2, \\ 1, & p \text { odd }, p \in P^{2}, \\ 2, & p \text { odd, } p \in P^{2} .\end{cases}
$$

Hence $G^{*}$ contains a cyclic subgroup $H$ of order $p^{e}$. Since the KrullSchmidt theorem is assumed valid for $R G^{*}$-modules, it also holds for $R H$-modules. Note that $p^{e} \in P^{2}$, and if $p=2$, then $p^{e} \in 2 P$.

Let $I$ denote the augmentation ideal of $R H$, so that

$$
I=\sum_{h \in H} R(h-1)
$$

Then

$$
\bar{I}=\sum_{h \in H} \bar{R}(h-1) \cong \bar{R}[x] /(x-1) p^{e-1},
$$

where the generator of the cyclic group $H$ acts on the right-hand module as multiplication by $x$. This shows that $\bar{I}$ is indecomposable, whence so is $I$.

We shall show next that if $K$ is a proper subgroup of $H$, and $\bar{M}$ is any $\bar{R} K$-module, then $\bar{I} \mid \bar{M}^{H}$ is impossible. For if such a relation were true, we could assume without loss of generality that $\bar{M}$ is an indecomposable $\bar{R} K$-module. Since $K$ is cyclic, the indecomposable $\bar{R} K$ modules may be listed explicitly, and have $K$-dimensions $1,2, \cdots$, $[K: 1]$. If $\bar{M}$ has dimension $[K: 1]$, then $\bar{M}=\bar{R} K$, and in that case $\bar{I} \mid \bar{R} H$. This is impossible since $\bar{R} H$ is indecomposable. On the other hand, if $\operatorname{dim} \bar{M}<[K: 1]$, then $\operatorname{dim} \bar{M}^{H}=[H: K] \cdot \operatorname{dim} \bar{M}<p^{e}-1$ $=\operatorname{dim} \bar{I}$, so also in this case $\bar{I}$ cannot be a direct summand of $\bar{M}^{H}$. As in [4], define the $R H$-modules $X$ and $Y$ by 


$$
X=\pi \cdot R H+I, \quad Y=\pi \cdot R H+R \cdot \sum_{h \in H} h .
$$

Then $X$ is a nonsplit extension of the factor module $R$ (with trivial action of $H$ ) by the submodule $I$, and hence $X$ is indecomposable. On the other hand, we showed in [4] that $Y$ is an extension of $R$ by a submodule $J$, where $\bar{R} \mid \bar{J}$. From these facts we were able to conclude that

$$
\bar{X} \cong \bar{Y} \cong \bar{R} \oplus \bar{I}, \quad X \text { not isomorphic to } Y .
$$

Furthermore, there exist exact sequences

$$
\begin{aligned}
& 0 \rightarrow X \rightarrow R H \rightarrow \bar{R} \rightarrow 0 \\
& 0 \rightarrow Y \rightarrow R H \rightarrow \bar{I} \rightarrow 0 .
\end{aligned}
$$

Let $r$ be a positive integer such that $p \nmid r$, and let $M$ be any $R H$ module. Define a new $R H$-module $M_{r}$ consisting of the same elements as $M$, but with a different action of $H$, namely, an element $h \in H$ acts on $M_{r}$ in the same way that $h^{r}$ acts on the original module $M$. Clearly, if $M$ is an extension of $A$ by $B$, then $M_{r}$ is an extension of $A_{r}$ by $B_{r}$. Further, if $R \mid M$ then also $R \mid M_{r}$.

We claim that $X \cong Y_{r}$ is impossible. To prove this, note first of all that $Y_{r}$ is an extension of $R$ by $J_{r}$. If $X \cong Y_{r}$ then $I \cong J_{r}$, and so $\bar{I} \cong \bar{J}_{r}$. This cannot hold true because $\bar{R} \mid \bar{J}$, so that $\bar{R} \mid \bar{J}_{r}$, while on the other hand $\bar{R} \nmid \bar{I}$. We have thus established our claim.

3. Proof of the main result. Keeping the notation of the preceding section, we are now ready to prove Theorem 2. Let us define $U=X^{G}$, $V=Y^{G}$. Since $(R H)^{G} \cong R G$, from the exact sequences in (1) we obtain a new pair of exact sequences

$$
\begin{aligned}
& 0 \rightarrow U \rightarrow R G \rightarrow \bar{R}^{G} \rightarrow 0, \\
& 0 \rightarrow V \rightarrow R G \rightarrow \bar{I}^{G} \rightarrow 0 .
\end{aligned}
$$

Since $\bar{X} \cong \bar{Y}$ we conclude that $\bar{U} \cong \bar{V}$.

For any $R G$-module $M$ of $R$-rank $m$, there is an exact sequence

$$
0 \rightarrow U \otimes_{R} M \rightarrow R G \oplus \cdots \oplus R G \rightarrow \bar{R}^{G} \otimes_{\bar{R}} \bar{M} \rightarrow 0,
$$

where $m$ summands occur in the center module. This implies by Schanuel's Lemma that the module $U \otimes_{R} M$ depends only upon $\bar{M}$. Hence we may conclude that

$$
U \otimes_{R} U \cong U \otimes_{R} V \cong V \otimes_{R} U \cong V \otimes_{R} V,
$$

and therefore that $\{U\}-\{V\}$ has square zero in $A(R G)$. 
In order to complete the proof of Theorem 2, it suffices to show that $\{U\}-\{V\}$ is nonzero, that is, that $U$ and $V$ are not isomorphic. Suppose that $U \cong V$, so that $X^{G} \cong Y^{G}$. Then

$$
\left(X^{\sigma}\right)_{H} \cong\left(Y^{\sigma}\right)_{H} \quad \text { as } R H \text {-modules. }
$$

However, $X \mid\left(X^{G}\right)_{H}$, and so also $X \mid\left(Y^{G}\right)_{H}$. Let us apply Mackey's Subgroup Theorem to the module $\left(Y^{G}\right)_{H}$. This yields

$$
\left(Y^{G}\right)_{H} \cong \sum_{0}^{\oplus}(g \otimes Y)_{K}{ }^{H}
$$

In this formula, $g$ ranges over a full set of representatives of the $(H, H)$-double cosets of $G$. For each such $g, K$ is the subgroup of $H$ given by $K=H \cap g \mathrm{Hg}^{-1}$. The $R K$-module $g \otimes Y$ is a subspace of $R G \otimes Y$, and the action of $K$ on $g \otimes Y$ is given by

$$
g h g^{-1}(g \otimes y)=g \otimes h y, \quad h \in H, \quad y \in Y .
$$

Since $X \mid\left(Y^{G}\right)_{H}$ and $X$ is indecomposable, we conclude that $X \mid(g \otimes Y)_{K}{ }^{H}$ for some $g$, and hence that $\bar{X} \mid(g \otimes \bar{Y})_{K}{ }^{H}$. By the remarks in $\S 2$, this cannot occur if $K$ is a proper subgroup of $H$. On the other hand, suppose that $K=H$, so that $g \mathrm{Hg}^{-1}=H$. If $h$ is a generator of the cyclic group $H$, we may write $g^{-1} h g=h^{r}$, where $p \nmid r$. Then the $\bar{R} H$-modules $g \otimes \bar{Y}$ and $\bar{Y}_{r}$ are isomorphic, and if $\bar{X} \mid(g \otimes \bar{Y})$, then $\bar{X} \cong \bar{Y}_{r}$. This is impossible by the results of $\S 2$. We have thus shown that $U$ and $V$ are nonisomorphic, which completes the proof of the theorem.

COROLlaRy. Let $R_{0}$ be a valuation ring in an algebraic number field, with maximal ideal $P_{0}$. Suppose that $G$ contains a cyclic sub-group $G^{*}$ of order $n$, where $n \in P_{0}^{2}$, and if $2 \in P_{0}$, assume further that $n \in 2 P_{0}$. Then $A\left(R_{0} G\right)$ contains at least one nonzero nilpotent element.

Proof. Even though the Krull-Schmidt theorem may not hold for $R_{0} G$-modules, we may nevertheless form the representation ring $A\left(R_{0} G\right)$ defined as above. If $L$ and $L^{\prime}$ are $R_{0} G$-modules, it is easily seen that $\{L\}=\left\{L^{\prime}\right\}$ in $A\left(R_{0} G\right)$ if and only if there exists an $R_{0} G$ module $M$ such that $L \oplus M \cong L^{\prime} \oplus M$.

The construction given in Theorem 2, with $R$ replaced by $R_{0}$, yields a pair of $R_{0} G$-modules $U_{0}, V_{0}$ such that $U=R \otimes U_{0}, V=R \otimes V_{0}$. However, the map $A\left(R_{0} G\right) \rightarrow A(R G)$ defined by $L \rightarrow R \otimes_{R_{0}} L$ is a monomorphism. Therefore $\left\{U_{0}\right\}-\left\{V_{0}\right\}$ is a nonzero nilpotent element of $A\left(R_{0} G\right)$, and the Corollary is proved. 


\title{
REFERENCES
}

1. C. W. Curtis and I. Reiner, Representation theory of finite groups and associative algebras, Interscience, New York, 1962.

2. J. A. Green, A transfer theorem for modular representations, J. of Algebra 1 (1964), 73-84.

3. M. F. O'Reilly, On the semisimplicity of the modular representation algebra of a finite group, Illinois J. Math. 9 (1965), 261-276.

4. I. Reiner, The integral representation ring of a finite group, Michigan Math. J. 12 (1965), 11-22.

UNIVERSITY OF ILLINOIS

\section{NOTE ON ANALYTICALLY UNRAMIFIED SEMI-LOCAL RINGS}

\author{
LOUIS J. RATLIFF, JR. ${ }^{1}$
}

All rings in this paper are assumed to be commutative rings with a unit element. If $B$ is an ideal in a ring $R$, the integral closure $B_{a}$ of $B$ is the set of elements $x$ in $R$ such that $x$ satisfies an equation of the form $x^{n}+b_{1} x^{n-1}+\cdots+b_{n}=0$, where $b_{i} \in B^{i}(i=1, \cdots, n)$. An ideal $B$ in $R$ is semi-prime in case $B$ is an intersection of prime ideals. If $R$ is an integral domain, then $R$ is normal in case $R$ is integrally closed in its quotient field. If $R$ is a semi-local (Noetherian) ring, then $R$ is analytically unramified in case the completion of $R$ (with respect to the powers of the Jacobson radical of $R$ ) contains no nonzero nilpotent elements.

Let $R$ be a semi-local ring with Jacobson radical $J$, and let $R^{*}$ be the completion of $R$. In [2], Zariski proved that if $R$ is a normal local integral domain, and if there is a nonzero element $x$ in $J$ such that $p R^{*}$ is semi-prime, for every prime divisor $\mathfrak{p}$ of $x R$, then $R$ is analytically unramified. In $[1$, p. 132] Nagata proved that if $R$ is a semilocal integral domain, and if there is a nonzero element $x$ in $J$ such that, for every prime divisor $\mathfrak{p}$ of $x R, \mathfrak{p} R^{*}$ is semi-prime and $R_{\mathfrak{p}}$ is a valuation ring, then $R$ is analytically unramified. (The condition $R_{\mathfrak{p}}$ is a valuation ring holds if $R$ is normal.) The main purpose of this note is to extend Nagata's result to the case where $R$ is a semi-local ring (Theorem 1). This extension will be given after first proving a

Received by the editors March 29, 1965.

1 Work on this paper was supported in part by the National Science Foundation. Grant GP3595. 